\title{
OPTIMAL FREQUENCY SWEEP METHOD IN MULTI-RATE CIRCUIT SIMULATION
}

\author{
KAI BITTNER* AND HANS GEORG BRACHTENDORF*
}

\begin{abstract}
.
Purpose - RF circuits often possess a multi-rate behavior. Slow changing baseband signals and fast oscillating carrier signals often occur in the same circuit. Frequency modulated signals pose a particular challenge.

Design/methodology/approach - The ordinary circuit differential equations (ODEs) are first rewritten by a system of (multi-rate) partial differential equations (MPDEs) in order to decouple the different time scales. For an efficient simulation we need an optimal choice of a frequency dependent parameter. This is achieved by an additional smoothness condition.

Finding - By incorporating the smoothness condition into the discretization, we obtain a nonlinear system of equations complemented by a minimization constraint. This problem is solved by a modified Newton method, which needs only little extra computational effort. The method is tested on a Phase Locked Loop with a frequency modulated input signal.

Originality/value - A new optimal frequency sweep method was introduced, which will permit a very efficient simulation of multi-rate circuits.
\end{abstract}

Key words. RF circuit simulation, multi-rate simulation, envelope simulation.

Paper type: Research paper

1. Introduction. Widely separated time-scales occur in many radio-frequency (RF) circuits such as mixers, oscillators, PLLs, etc., making the analysis with standard numerical methods difficult and costly. Low frequency or baseband signals and high frequency carrier signals often occur in the same circuit, enforcing very small time-steps over a long timeperiod for the computation of the numerical solution. The occurrence of widely separated time-scales is also referred to as a multi-scale or multi-rate problem, where classical numerical techniques require prohibitively long run-times.

A method to circumvent this bottleneck is to reformulate the ordinary circuit equations, which are differentieal algebraic equations (DAEs), as a system of partial DAEs (multi-rate PDAE). The method was first presented in (Brachtendorf 1994, Brachtendorf, Welsch, Laur and Bunse-Gerstner 1996), specialized to compute steady states.The technique was adapted to the transient simulation of driven circuits with a-priori known frequencies in (Ngoya and Larchevèque 1996, Roychowdhury 1997). A generalization for circuits with a-priori unknown or time-varying frequencies was developed in (Brachtendorf 1997, Brachtendorf and Laur 1998a, Brachtendorf 2001). This approach opened the door to multi-rate techniques with frequency modulated signal sources or autonomous circuits such as oscillators with a priori unknown fundamental frequency (Brachtendorf and Laur 1998a, Brachtendorf and Laur 1998b, Brachtendorf 2001, Houben 2004, Pulch 2008b, Pulch 2008a).

Here, we present a new approach for the computation of a not a-priori known, timevarying frequency, which is driven by the requirement to have a smooth multi-rate solution, crucial for the efficiency of the computation.

2. The multi-rate circuit simulation problem. We consider circuit equations in the charge/flux oriented modified nodal analysis (MNA) formulation, which yields a mathematical model in the form of a system of differential-algebraic equations (DAEs):

$$
\frac{d}{d t} q(x(t))+g(x(t))=s(t) .
$$

${ }^{*}$ University of Applied Sciences Upper Austria, Softwarepark 11, 4232 Hagenberg, Austria 
Here $x(t) \in \mathbb{R}^{n}$ is the vector of node potentials and specific branch voltages and $q(x) \in \mathbb{R}^{n}$ is the vector of charges and fluxes. The vector $g(x) \in \mathbb{R}^{n}$ comprises static contributions, while $s(t) \in \mathbb{R}^{n}$ contains the contributions of independent sources.

To separate different time scales the problem is reformulated as a multi-rate PDAE (cf. (Brachtendorf 2001, Houben 2004, Pulch 2008a, Pulch 2008b)), i.e.,

$$
\frac{\partial}{\partial \tau} q(\hat{x}(\tau, t))+\omega(\tau) \frac{\partial}{\partial t} q(\hat{x}(\tau, t))+g(\hat{x}(\tau, t))=\hat{s}(\tau, t) .
$$

If the new source term is chosen, such that

$$
s_{\theta}(t)=\hat{s}\left(t, \Omega_{\theta}(t)\right)
$$

where $\Omega_{\theta}(t)=\theta+\int_{0}^{t} \omega(s) d s$, then a solution $\hat{x}$ of 2.2 determines a family $\left\{x_{\theta}: \theta \in \mathbb{R}\right\}$ of solutions for

$$
\frac{d}{d t} q(x(t))+g(x(t))=s_{\theta}(t)
$$

by

$$
x_{\theta}(t)=\hat{x}\left(t, \Omega_{\theta}(t)\right) .
$$

Although the formulation (2.2) is valid for any circuit, it offers a more efficient solution only for certain types of problems. This is the case if $\hat{x}(\tau, t)$ is periodic in $t$ and smooth with respect to $\tau$. Then, a semi-discretization with respect to $\tau$ can be done resulting in a relative small number of periodic boundary problems in $t$ for only a few discretization points $\tau_{\ell}$. In the sequel we will consider 2.2 with periodicity conditions in $t$, i.e., $\hat{x}(\tau, t)=\hat{x}(\tau, t+P)$ and suitable initial conditions $\hat{x}(0, t)=X_{0}(t)$. Here $P$ is an arbitrary but fixed period length, which results in a scaling of $\hat{x}(\tau, t)$ and $\omega(\tau)$ as we will see in the next section.

Note that for a given initial value for the original circuit equations 2.1 the multi-rate formulation 2.2. is not unique. First it does not correspond to a single problem, but to a family of problems as described in (2.4). This usually permits some freedom in the choice of the multi-rate source term $\hat{s}(\tau, t)$ and for the initial conditions. Furthermore, $\omega(\tau)$ can, in principle, be chosen arbitrarily, but influences on the other hand, which choice of $\hat{s}(\tau, t)$ satisfies 2.3.

In the sequel, we want to study how the above freedom can be used to facilitate an efficient numerical solution of 2.2. The smoothness of $\hat{x}(\tau, t)$ is essential for the efficiency of classical solvers. That is, the freedom we have for the formulation of 2.2 should be used to make the solution as smooth as possible.

3. Meaning and suitable choice of $\omega(\tau)$. It can be easily verified that $\omega(\tau)$ can be any positive function, if the source term $\hat{s}(\tau, t)$ is chosen to satisfy 2.3). Therefore, we will first investigate, what effect different selections of $\omega(\tau)$ have. Let $\omega_{1}(\tau)$ and $\omega_{2}(\tau)$ be two choices. If $\hat{x}_{1}(\tau, t)$ and $\hat{x}_{2}(\tau, t)$ both satisfy 2.5 , then it is obvious that

$$
\hat{x}_{2}(\tau, t)=\hat{x}_{1}(\tau, t+S(\tau)),
$$

where $S(\tau)=\int_{0}^{\tau} \omega_{1}(s)-\omega_{2}(s) d s$. That is, for a fixed $\tau$ changing $\omega(t)$ results in a phase shift of $\hat{x}(\tau, t)$. A corresponding phase shift for the source term has to be performed in order to satisfy 2.3 . The following Lemma describes how $\omega(\tau)$ affects the smoothness of $\hat{x}(\tau, t)$.

LEMMA 3.1. Let $L>\frac{P}{\omega(\tau)}$ for any $\tau>0$. If

$$
\|\hat{x}(\tau+\delta, t)-\hat{x}(\tau, t)\| \leq \varepsilon,
$$


for $\delta \in(0, L]$, then

$$
\left\|x_{\theta}(\tau+T(\tau))-x_{\theta}(\tau)\right\| \leq \varepsilon,
$$

where $T(\tau)$ is the unique solution of $P=\int_{\tau}^{\tau+T(\tau)} \omega(s) d s$.

Proof. Following 2.5 we have

$$
\begin{aligned}
x_{\theta}(\tau+T(\tau)) & =\hat{x}\left(\tau+T(\tau), \theta+\int_{0}^{\tau+T(\tau)} \omega(s) d s\right) \\
& =\hat{x}\left(\tau+T(\tau), \theta+\int_{0}^{\tau} \omega(s) d s+P\right) \\
& =\hat{x}\left(\tau+T(\tau), \theta+\int_{0}^{\tau} \omega(s) d s\right) .
\end{aligned}
$$

By the mean value theorem for integration we obtain

$$
P=T(\tau) \omega(\xi), \quad \xi \in(\tau, \tau+T(\tau)) .
$$

Thus, $T(\tau)<L$ and $(3.3)$ follows from 3.2 by 2.5 . .

Lemma 3.1 states that we can only expect smoothness of $\hat{x}(\tau, t)$ in $\tau$ if $x_{\theta}$ is nearly $T(\tau)$-periodic in a neighborhood of $\tau$.

Since typical multi-rate signals behave locally like a periodic signal, i.e., $x_{\theta}(t) \approx$ $x_{\theta}\left(t+T\left(t^{*}\right)\right)$ as long as $t$ is close to some $t^{*}$. That is, there should be a choice of $\omega(\tau)$ such that $\hat{x}\left(\tau_{1}, t\right)$ and $\hat{x}\left(\tau_{2}, t\right)$ do not differ much for sufficiently small $\tau_{2}-\tau_{1}$. This leads to the additional condition

$$
\int_{0}^{P}\left|\frac{\partial}{\partial \tau} \hat{x}(\tau, t)\right|^{2} d t \rightarrow \min
$$

in order to determine $\omega(\tau)$. A similar condition is used in (Pulch 2008b)). The difference to our approach is in the discretization of the problem. While Pulch derives a phase condition from the minimization condition, we will discretize the minimization condition directly, which will lead to a smaller linear system to be solved in the algorithm. Furthermore, we are using a Rothe method for the discretization of the PDAE, which allows a completely adaptive solution.

A different approach is suggested in (Houben 2004), where $\hat{x}(\tau, t)$ is replaced by $q(\hat{x}(\tau, t))$ in 3.4). Although this seems the proper condition for an efficient discretization of the derivative in $\tau$ in the multi-rate PDAE [2.2), another problem occurs here. Using an implicit multistep method for the discretization in $\tau$, we need a predictor (as initial guess for Newton's method), which is usually based on the solution of the previous time step. Here, a condition on $\hat{x}(\tau, t)$ as in 3.4 occurs naturally. A condition on $q(\hat{x}(\tau, t))$ covers only contributions of the signal which are already smoothed (by capacitances or inductances), and might not provide a sufficient initial guess.

It turns out that condition 3.4 yields the expected result for amplitude and frequency modulation of a periodic signal, i.e., for typical multirate RF signals.

LEMMA 3.2. Assume $x_{\theta}(t)=a(t) y(\theta+\tilde{\Omega}(t))$ with $y(t)=y(t+P)$ is a solution of 2.4 with non-trivial $a(t)$ and $y^{\prime}(t)$. The solution of the corresponding multi-rate problem 2.2 satisfies 3.4 if and only if $\omega(\tau)=\tilde{\Omega}^{\prime}(\tau)$ and $\hat{x}(\tau, t)=a(\tau) y(t)$.

Proof. According to 2.5) and (3.1) we can write the multi-rate solution as $\hat{x}(\tau, t)=$ $a(\tau) y(t+S(\tau))$, where $S(\tau)$ vanishes if and only if $\omega(\tau)=\tilde{\Omega}^{\prime}(\tau)$. Taking the derivative with respect to $\tau$ we obtain

$$
\frac{\partial}{\partial \tau} \hat{x}(\tau, t)=a^{\prime}(\tau) y(t+S(\tau))+a(\tau) y^{\prime}(t+S(\tau)) S^{\prime}(\tau) .
$$


Note, that due to periodicity of $y(t)$ we have

$$
\int_{0}^{P} y(t+S(\tau)) y^{\prime}(t+S(\tau)) d t=\frac{1}{2} \int_{0}^{P} \frac{d}{d t} y^{2}(t) d t=\frac{1}{2}\left(y^{2}(P)-y^{2}(0)\right)=0 .
$$

Thus, the expression in (3.4), which has to be minimized becomes

$$
\left(a^{\prime}(\tau)\right)^{2} \int_{0}^{P} y^{2}(t) d t+\left(a(\tau) S^{\prime}(\tau)\right)^{2} \int_{0}^{P}\left(y^{\prime}(t)\right)^{2} d t .
$$

Obviously the expression becomes minimal if $S^{\prime}(\tau)=0, \tau \in\left(\tau_{1}, \tau_{2}\right]$. From 3.1 we see that $S(0)=0$ and the statement is proven. $\square$

Applying the approach to more general problems, permits to consider $\frac{\omega(t)}{P}$ as a generalization of the instantaneous frequency of the multi-rate problem, if (3.4) is satisfied.

For the numerical solution of the multi-rate problem we have to discretize the MPDAE's (2.2) together with the smoothness condition (3.4). The following section presents a new approach for the numerical solution of the multi-rate problem with unknown frequency parameter.

4. Discretization. We discretize (2.2) with respect to $\tau$ by a Rothe method using a linear multi step method and obtain

$$
\sum_{i=0}^{s}\left\{\alpha_{i}^{k} q\left(X_{k-i}(t)\right)+\beta_{i}^{k}\left(\omega_{k-i} \frac{d}{d t} q\left(X_{k-i}(t)\right)+g\left(X_{k-i}(t)\right)-\hat{s}\left(\tau_{k-i}, t\right)\right)\right\}=0
$$

complemented by the periodicity condition $X_{k}(t)=X_{k}(t+P)$. Here we have to determine an approximation $X_{k}(t)$ of $\hat{x}\left(\tau_{k}, t\right)$ from known, approximative solutions $X_{k-i}(t)$ at previous time steps $\tau_{k-i} . i=1, \ldots, s$. Examples are the trapezoidal rule with $s=1, \alpha_{i}^{k}=1$ and $\beta_{i}^{k}=\frac{2}{\tau_{k}-\tau_{k-1}}$ or GEAR-BDF of order $s$ with $\beta_{i}^{k}=\delta_{i, 0}$ and suitable $\alpha_{i}^{k}$. That is, $X_{k}$ is the solution of a periodic boundary value problem for the DAEs given in 4.1.

Assuming that the $\tau_{k-i}, \omega_{k-i}$ and $X_{k-i}(t)$ are known and fixed for $i>0$ we define

$$
\begin{aligned}
& f_{k}(x, t):=\alpha_{0}^{k} q(x)+\beta_{0}^{k}\left(g(x)-\hat{s}\left(\tau_{k}, t\right)\right) \\
& \quad+\sum_{i=1}^{s}\left\{\alpha_{i}^{k} q\left(X_{k-i}(t)\right)+\beta_{i}^{k}\left(\omega_{k-i} \frac{d}{d t} q\left(X_{k-i}(t)\right)+g\left(X_{k-i}(t)\right)-\hat{s}\left(\tau_{k-i}, t\right)\right)\right\}
\end{aligned}
$$

as well as $q_{k}(x):=\beta_{0}^{k} q(x)$. Then 4.1 becomes the periodic boundary value problem

$$
\omega \frac{d}{d t} q_{k}(x(t))+f_{k}(x(t), t)=0, \quad x(t)=x(t+P) .
$$

Furthermore, $\omega_{k}$ is an approximation for $\omega\left(\tau_{k}\right)$, which has to be determined during the computation (if not known in advance). Since the midpoint rule states for a suitable choice of $\tau \in\left(\tau_{1}, \tau_{2}\right)$ that

$$
\frac{\partial}{\partial \tau} \hat{x}(\tau, t)=\hat{x}\left(\tau_{2}, t\right)-\hat{x}\left(\tau_{1}, t\right)
$$

we replace condition 3.4 by

$$
\int_{0}^{P}\left|X_{k}(t)-X_{k-1}(t)\right|^{2} d t \rightarrow \min
$$


i.e., we aim to minimize the change of $X_{k}(t)$ from one time step to the next, which corresponds well to the original goal.

If we have a smooth envelope, we may be able to choose the step sizes $\tau_{k}-\tau_{k-1}$ much larger than the period $T(\tau)=\frac{P}{\omega(\tau)}$. That means the number of time steps will become much smaller compared to the number of oscillations of the carrier signal. This can lead to an essential gain in computation time compared to transient analysis, since the computation cost for one period is comparable to one envelope time step.

The periodic problem (4.2) can be solved by a collocation or Galerkin method, where $X_{k}(t)$ is expanded in a periodic basis $\left\{\phi_{k}\right\}$ (as a Fourier, B-spline, or wavelet basis) and tested at collocation points or integrated against test functions (see e.g. (Bittner and Dautbegovic 2012b, Bittner and Dautbegovic 2012a)). This leads to a nonlinear system

$$
F(\boldsymbol{c}, \omega)=0
$$

of equations for the coefficients $\boldsymbol{c}=\boldsymbol{c}^{(k)}=\left(c_{\ell}^{(k)}\right)_{\ell}$ of the basis expansion $X_{k}(t)=$ $\sum_{\ell} c_{\ell}^{(k)} \phi_{\ell}(t)$ and the unknown frequency parameter $\omega$. Here, condition 4.3 is replaced by a condition on the expansion coefficients, namely

$$
\sum_{\ell}\left\|c_{\ell}^{(k)}-c_{\ell}^{(k-1)}\right\|_{2}^{2} \rightarrow \min
$$

This means, that we are still minimizing the distance of $X_{k}(t)$ and $X_{k-1}(t)$, although with respect to a slightly modified measure.

The nonlinear system 4.4 is solved by a modified Newton's method. The linearization of the problem yields the under-determined system

$$
\boldsymbol{A} \boldsymbol{d}_{c}+d_{\omega} \boldsymbol{z}=\boldsymbol{b}
$$

with the right hand site $\boldsymbol{b}=F(\boldsymbol{c}, \omega)$, the Jacobian $\boldsymbol{A}=\frac{\partial}{\partial \boldsymbol{c}} F(\boldsymbol{c}, \omega)$, and $\boldsymbol{z}=\frac{\partial}{\partial \omega} F(\boldsymbol{c}, \omega)$. Furthermore, $\boldsymbol{d}_{c}$ and $d_{\omega}$ are the updates in the Newton iteration

$$
\boldsymbol{c}^{(k, j)}=\boldsymbol{c}^{(k, j-1)}-\boldsymbol{d}_{c}, \quad \omega_{k, j}=\omega_{k, j-1}-d_{\omega},
$$

with an initial guess chosen as $\boldsymbol{c}^{(k, 0)}=\boldsymbol{c}^{(k-1)}$ and $\omega_{k, 0}=\omega_{k-1}$.

To find a solution satisfying 4.5 we rewrite 4.6 as

$$
\boldsymbol{d}_{c}=\tilde{\boldsymbol{b}}-d_{\omega} \tilde{\boldsymbol{z}},
$$

where $\tilde{b}=A^{-1} b$ and $\tilde{z}=A^{-1} \boldsymbol{z}$ are computed by solving the corresponding linear systems. This needs a LU-decomposition of $\boldsymbol{A}$ and two forward-backward substitution, i.e., the computational costs are only slightly higher as for $\omega(\tau)$ fixed in advance. Possible higher step sizes and faster convergence of Newton's method justify this extra cost if a good choice for $\omega(\tau)$ is not available in advance. In the $j$-th Newton step $\boldsymbol{c}^{(k, j)}=\boldsymbol{c}^{(k, j-1)}-\boldsymbol{d}_{c}$ we determine the Newton correction, which satisfies 4.5 ) as follows. We want

$$
\left\|\boldsymbol{c}^{(k, j)}-\boldsymbol{c}^{(k-1)}\right\|_{2}^{2}=\|\underbrace{\boldsymbol{c}^{(k, j-1)}-\boldsymbol{c}^{(k-1)}-\tilde{\boldsymbol{b}}}_{\boldsymbol{a}}+d_{\omega} \tilde{\boldsymbol{z}}\|_{2}^{2}
$$

to be minimal. Obviously the minimum is attained for $d_{\omega}=-\frac{\tilde{z}^{T} \boldsymbol{a}}{\tilde{\boldsymbol{z}}^{T} \tilde{\boldsymbol{z}}}$. 
5. Numerical test - A phase locked loop. The method has been tested on several circuits. We show results from the multi-rate simulation of a Phase Locked Loop (PLL) with a frequency modulated input signal. The phase of the output signal is synchronized with the phase of the input "reference" signal. This is achieved, by comparing the phases of the "reference" signal and the output or "feedback" signal in a phase detector. The result is then filtered, in order to stabilize the behavior of the PLL, and fed to a Voltage Controlled Oscillator (VCO) whose frequency depends on the (filtered) phase difference. The output is fed back (possibly through a frequency divider) to the phase detector. If the PLL is locked, the phases of reference and feedback signal are synchronized. Thus both signal will have (almost) the same local frequency.
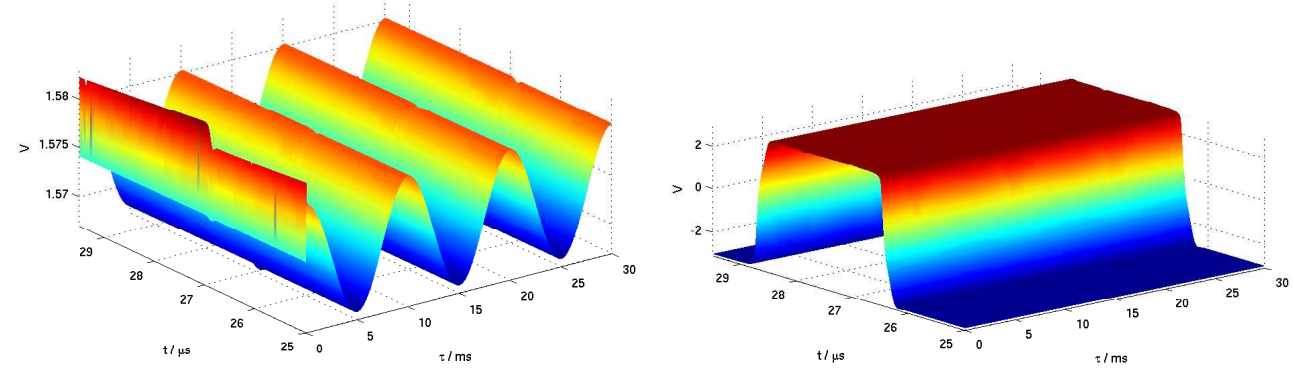

FIG. 5.1. Control and feedback signal of the PLL

We have tested a PLL (containing 145 MOSFETs and 80 unknowns) with a frequency modulated sinusoidal signal with frequency $222 \mathrm{kHz}$. The baseband signal is also sinusoidal with frequency $200 \mathrm{~Hz}$ and frequency deviation $200 \mathrm{~Hz}$. We have chosen this example, since it was particular challenging, due to its size, but also due its digital-like behavior, which requires an efficient adaptive representation of the solution, as well as a very accurate estimate of the frequency parameter $\omega(\tau)$. Although the baseband signal is known to us after locking of the PLL, this information is not provided to the algorithm. This permits a verification of the $\omega(\tau)$ determined by our algorithm. Before the locking of the PLL the optimal $\omega(\tau)$ is not known to us, and the estimate of the algorithm proved essential for this part of the simulation to be successful.

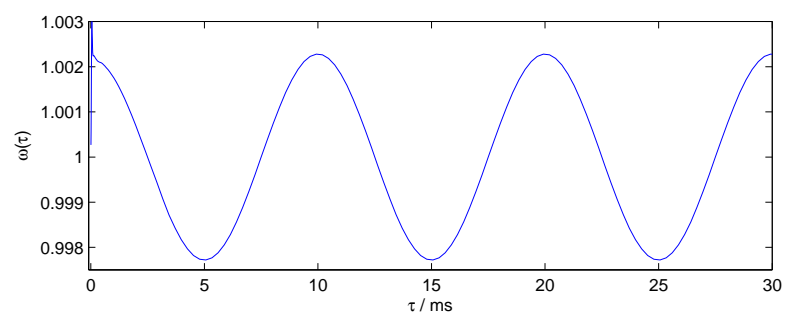

FIG. 5.2. Frequency parameter $\omega(\tau)$

Due to the chosen circuit design most of the signals show a digital behavior such that the solution can not efficiently be expanded by a Fourier basis. Thus, using the Harmonic Balance method is not suited for the solution of the problem, in particular since the use of a frequency divider results in a wide frequency range of the solution. Therefore, the periodic problem is solved by an adaptive spline wavelet method described in (Bittner and Dautbegovic 2012b, Bittner and Dautbegovic 2012a). The quality of the estimate of $\omega(\tau)$ is 
essential for the efficiency of the method, because this results in a good initial guess for the solution and the spline grid in Newton's method.

In Figure 5.1 (left) one can see the control signal for the VCO (the filtered output of the phase detector), which controls the frequency of the oscillator. Note, that after the locking phase at the beginning the envelope corresponds to the baseband signal, while the carrier signal is due to the filtering almost constant. Figure 5.1 (right) shows the digital feedback signal. Note that $\omega(\tau)$ was chosen to satisfy the condition (4.5). The plot of $\omega(\tau)$ in Figure 5.2 fits almost perfectly the baseband signal, after the PLL is locked. In the light of Lemma 3.2. the graphs in Figures 5.1 and 5.2 show indeed a frequency modulated box shaped signal. The optimal choice of $\omega(\tau)$ results in high smoothness with respect to $\tau$. This allows to do the shown envelope simulation using only 74 envelope time steps, while a corresponding transient analysis would contain 3100 oscillations. The wavelet envelope simulation of this circuit was done in $7 \mathrm{~min}$. However, a comparable transient simulation on the same simulator needed 33 hours.

6. Conclusion. We have presented a new method for an accurate estimate of an unknown frequency parameter in multi-rate envelope simulations, which permits an efficient simulation even for challenging problems.

\section{REFERENCES}

K. Bittner and E. Dautbegovic (2012a), Adaptive wavelet-based method for simulation of electronic circuits, in Scientific Computing in Electrical Engineering 2010 (B. Michielsen and J.-R. Poirier, eds), Mathematics in Industry, Springer, Berlin Heidelberg, pp. $321-328$.

K. Bittner and E. Dautbegovic (2012b), Wavelets algorithm for circuit simulation, in Progress in Industrial Mathematics at ECMI 2010 (M. Günther, A. Bartel, M. Brunk, S. Schöps and M. Striebel, eds), Mathematics in Industry, Springer, Berlin Heidelberg, pp. 5 - 11.

H. G. Brachtendorf (1994), Simulation des eingeschwungenen Verhaltens elektronischer Schaltungen, Shaker, Aachen.

H. G. Brachtendorf (1997), On the relation of certain classes of ordinary differential algebraic equations with partial differential algebraic equations, Technical Report 1131G0-971114-19TM, Bell-Laboratories.

H. G. Brachtendorf (2001), 'Theorie und Analyse von autonomen und quasiperiodisch angeregten elektrischen Netzwerken. Eine algorithmisch orientierte Betrachtung', Universität Bremen. Habilitationsschrift.

H. G. Brachtendorf and R. Laur (1998a), A time-frequency algorithm for the simulation of the initial transient response of oscillators, in Proc. IEEE Int. Symp. on Circuits and Systems, Monterey.

H. G. Brachtendorf and R. Laur (1998b), Transient simulation of oscillators, Technical Report 1131G0-98041009TM, Bell-Laboratories.

H. G. Brachtendorf, G. Welsch, R. Laur and A. Bunse-Gerstner (1996), 'Numerical steady state analysis of electronic circuits driven by multi-tone signals', Electronic Engineering 79(2), 103-112.

S. Houben (2004), Simulating multi-tone free-running oscillators with optimal sweep following, in Scientific Computing in Electrical Engineering 2010 (W. Schilders, E. ter Maten and S. Houben, eds), Mathematics in Industry, Springer, Berlin, pp. 240 - 247.

E. Ngoya and R. Larchevèque (1996), Envelope transient analysis: A new method for the transient and steady state analysis of microwave communication circuit and systems, in Proc. IEEE MTT-S Int. Microwave Symp., San Francisco, pp. 1365-1368.

R. Pulch (2008a), 'Initial-boundary value problems of warped mpdaes including minimisation criteria', Math. Comput. Simulat. 79, 117-132.

R. Pulch (2008b), 'Variational methods for solving warped multirate partial differential algebraic equations', SIAM J. Scient. Computing 31(2), 1016-1034.

J. Roychowdhury (1997), Efficient methods for simulating highly nonlinear multi-rate circuits, in Proc. IEEE Design Automation Conf., pp. 269-274. 